\title{
Cauda Equina Syndrome: presentation, outcome, and predictors with focus on micturition, defecation, and sexual dysfunction
}

\author{
N. S. Korse ${ }^{1}$ J. A. Pijpers ${ }^{2}$ E. van Zwet ${ }^{3} \cdot$ H. W. Elzevier ${ }^{4} \cdot$ \\ C. L. A. Vleggeert-Lankamp ${ }^{1}$
}

Received: 10 October 2016/Revised: 25 November 2016/ Accepted: 2 January 2017/Published online: 19 January 2017

(C) The Author(s) 2017. This article is published with open access at Springerlink.com

\begin{abstract}
Background Even though micturition, defecation, and sexual function are substantially affected in cauda equina syndrome (CES), data on outcome are scarce.

Methods Medical files of patients operated on lumbar herniated disc were screened for CES and retrospectively analyzed for baseline characteristics, outcome of micturition, defecation, and sexual function and possible predictors.

Results Seventy-five CES patients (52\% men) were included with a mean age of 44 years. L5-S1 was the most common affected level. Duration of CES complaints at presentation was, on average, $84 \mathrm{~h}$ (median $48 \mathrm{~h}$ ). Prevalence of symptoms at presentation: sciatica (97\%), altered sensation of the saddle area $(93 \%)$, micturition dysfunction (92\%), and defecation dysfunction (74\%). Only 26 patients were asked about sexual dysfunction of whom 25 patients experienced dysfunction. Female gender was associated with more defecation dysfunction at presentation than male gender (OR 4.11; $p=0.039$ ). All patients underwent decompressive surgery. Two post-operative follow-up (FU) moments took place after a mean of $75 \mathrm{~h}$ and 63 days.
\end{abstract}

$\triangle$ N. S. Korse

n.s.korse@lumc.nl

1 Department of Neurosurgery, Leiden University Medical Center, LUMC, Postzone J11-R-83, Postbus 9600, 2300 RC Leiden, The Netherlands

2 Department of Neurology, Leiden University Medical Center, Leiden, The Netherlands

3 Department of Medical Statistics, Leiden University Medical Center, Leiden, The Netherlands

4 Department of Urology, Leiden University Medical Center, Leiden, The Netherlands
Outcomes at second FU moment: micturition dysfunction $48 \%$, defecation dysfunction $42 \%$, sexual dysfunction $53 \%$, sciatica $48 \%$, and altered sensation of the saddle area $57 \%$. A shorter time to decompression was associated with more sciatica at FU $1(p=0.042)$ which effect had disappeared at FU 2.

Conclusion This study is unique in (1) displaying the presenting features in a large cohort of CES patients, (2) demonstrating that recovery after decompression is slow and far from complete in the majority of patients with regard to micturition, defecation, and sexual function and (3) evaluating predictors for outcome.

Keywords Cauda equina syndrome - Presentation . Outcome - Micturition · Defecation - Sexual dysfunction · Predictors

\section{Introduction}

Cauda equina syndrome (CES) is a neurological condition caused by compression of the cauda equina, most commonly described as a combination of sensory loss of the saddle area, motor deficit and/or loss of reflexes of the lower limbs, micturition dysfunction, defecation complaints, and/or sexual dysfunction [1, 2]. The first article about CES appeared in 1934, in which a combination of neurological and urological complaints in patients with a ruptured intervertebral disc was described [3]. A herniated disc is still the most common cause of cauda equina compression; in literature, $45 \%$ of cases of CES are attributed to a lumbar herniated disc [1].

In the last decades, especially the topic of timing of decompression and its relation to outcome has gained much attention in literature, with several small studies showing 
better-albeit not always significant-outcomes after the early decompression [4-9]. Other studies could not demonstrate a better outcome after early decompression $[10,11]$. The value of urgent decompression was most convincingly showcased in the meta-analysis of Ahn et al., showing a better prognosis of sensory, motor, urinary, and rectal function in patients being decompressed within $48 \mathrm{~h}$ of presentation, compared to a group being decompressed after $48 \mathrm{~h}$ [12]. These results were confirmed by others [13, 14].

In literature, there is a little focus on the prognosis of micturition, defecation, and sexual function [2]. This is remarkable considering the definition of CES. Recently, clinicians in spinal care were found to barely discuss sexual health and/or defecation at presentation and at follow-up, suggested to be due to, e.g., lack of knowledge or time $[2,15,16]$. Clinicians who do want to inform their patients about the prognosis of these functions are confronted with scarce data. This study was performed to (1) evaluate outcome of micturition, defecation, and sexual function in cauda equina syndrome after decompression and to (2) find possible predictors of outcome. In addition, presenting features of CES were analyzed.

\section{Materials and methods}

The medical records of patients operated in the Leiden University Medical Centre (LUMC; university hospital and referral centre for high complex spinal surgery) between January 1995 and September 2010 with the surgery code 'lumbar discectomy' or 'recurrent lumbar discectomy' were screened by two independent researchers (NSK, JAP) to identify patients with cauda equina syndrome (CES). Criteria to diagnose a patient with CES were, according to consensus of literature, one or more of the following: (1) dysfunction of micturition, defecation, and/or sexual function (not being attributable to use of opiates or previous disease), (2) altered sensation of the saddle area, with possible neurologic deficit in the lower limb (motor or sensory loss or reflex changes) [1,2]. Patients filed with a diagnosis of CES but not meeting those criteria were excluded. In case of doubt about the diagnosis of CES, a third assessor (CLAVL) was consulted. To check interobserver reliability between the two reviewers, $10 \%$ of cases were independently screened by both of them.

The following data were extracted from the medical file:

- Baseline characteristics (at presentation): Gender; Age; Level of herniated disc as stated in the file; Relevant medical history; Referring center (if applicable); Use of opiates and/or laxatives; Duration of complaints of herniated disc (defined by the presence of sciatica);
Duration of CES complaints; Information about micturition, defecation, sexual function, altered sensation of the saddle area, and/or sciatica; Information about anal sphincter reflex and anal sphincter tension.

- Surgery: Time between presentation at first doctor and decompression (time to decompression); Type of decompressive surgery.

- Follow-up: Information about micturition, defecation, sexual function, altered sensation of the saddle area, and/or sciatica at three follow-up moments: (1) At hospital discharge (FU 1). In case notes were taken several times during the first days post-operative, the last notes before discharge were used; (2) At the first outpatient visit, regularly planned 6 weeks post-operatively (FU 2); (3) At the second outpatient visit, which was not regularly planned (FU 3).

Data were collected in Excel and imported in SPSS.

\section{Statistical analyses}

Data were analyzed using SPSS Statistics 23.0 (SPSS Inc., Chicago, IL, USA). Demographic values and other patient characteristics were analyzed with frequencies. Investigating proportions between independent groups of categorical data was done with Pearson's Chi-squared test; Fisher's exact Test was used to compare groups with cell counts less than expected. For paired groups with categorical data, McNemar's test was used. Predictors for outcome and presentation were analyzed using a binary logistic regression model; in case of quasi-complete separation of the data, the concerning model was not run or the concerning predictor was removed from the model to maintain reliable models. Two-sided $p$ values $<0.05$ were considered statistically significant. In case of multiple testing, the Bonferroni method was used to correct $p$ values. Some numerical data were grouped together for analyses, e.g., timing of decompression was stratified into six groups: $\leq 12,13-24,25-36,37-48,49-72$, and $>72 \mathrm{~h}$.

\section{Missing data}

To run the regression models and for displaying outcome at FU 2, multiple imputation with five imputation sets was used for the following variables: duration of CES complaints; duration of complaints of herniated disc; time to decompression; micturition dysfunction at FU 1, 2 and 3; defecation dysfunction at presentation, FU 1, 2, and 3; altered sensation of the saddle area at FU 1, 2 and 3; sciatica at FU 1, 2 and 3. Multiple imputation was not performed for sexual dysfunction data due to scarcity of these data, to avoid bias. Pooled data (i.e. derived through multiple imputation) are presented as main data. Original data 
(not derived through multiple imputation) are presented as corrected (corrected for the number of patients for whom documentation is available at the concerning follow-up moment) and as raw (not corrected for the number of patients). Due to an anticipated high amount of loss to follow up at FU 3, outcome at FU 2 was defined as main outcome and data at FU 3 were not used for regression analysis. For the patients for whom data at FU 3 are available, this will be mentioned separately.

\section{Results}

In the period January 1995-September 2010, a total of 744 surgeries coded as '(recurrent) lumbar discectomy' were performed at LUMC, for a total of 696 patients: 38 patients had surgery twice, 10 patients had triple surgery. Out of 696 patients, 75 patients $(10.8 \%)$ were found to have CES. One female patient who underwent a lumbar discectomy twice, met CES criteria twice; however, since she had not recovered from her first CES completely, only the first surgery was included for analysis. Interobserver reliability regarding diagnosing CES was analyzed using Cohen's Kappa. There was substantial agreement between the two reviewers' judgement $(\kappa=0.635)$.

\section{Patient characteristics}

For patients characteristics; see Table 1 and Fig. 1. Since the LUMC serves as a referral hospital, the majority of included patients presented first at other hospitals (73.7\%). Thirty-two patients $(42.7 \%)$ used opiates at presentation. Use of laxatives was not significantly higher in the group using opiates: $25.0 \%$ of opiate positive patients used laxatives versus $19.5 \%$ of opiate negative patients $(p=0.574)$.

\section{Presenting features of CES}

Information about sciatica, altered sensation of the saddle area and micturition dysfunction, was available for all patients at presentation and was present in 97.3, 93.3 and $92.0 \%$, respectively (Fig. 2). Majority of sciatica was unilateral $(60.3 \%)$. Altered sensation of the saddle area was classified as either hypoesthesia (75.7\%), anesthesia (17.1\%), or dysthesia (7.1\%). Micturition dysfunction was classified as having an indwelling catheter $(39.1 \%)$, needing clean intermittent catheterization $(2.9 \%)$, documented residual of bladder $(5.3 \%)$, or subjective complaints, e.g., reduced feeling of passing urine or mild incontinence $(52.2 \%)$. Information about the presence of defecation dysfunction was available in 61 patients, of whom $73.8 \%$ had dysfunction, classified as any complaint of defecation
Table 1 Patient characteristics $(n=75)$

\begin{tabular}{ll}
\hline & $n(\%)$ \\
\hline Male & $39(52.0)$ \\
Mean age & $\begin{array}{c}\text { 43.6 years (SD 10.4, } \\
\text { range 27-78) }\end{array}$ \\
Level of disc lesion (as documented in file)* & \\
L1-L2 & $1(1.3)$ \\
L2-L3 & $3(3.8)$ \\
L3-L4 & $6(7.6)$ \\
L4-L5 & $29(36.7)$ \\
L5-S1 & $39(49.3)$ \\
L6-S1 & $1(1.3)$ \\
Origin of referral & \\
Neurologist LUMC & $15(20.0)$ \\
Emergency room LUMC & $2(2.7)$ \\
Other hospital in the area & $55(73.3)$ \\
General practitioner & $2(2.7)$ \\
Unknown & $1(1.3)$ \\
Use of opiates at presentation & \\
Yes & $32(42.7)$ \\
No & $41(54.6)$ \\
Unknown & $2(2.7)$ \\
Use of laxatives & \\
Yes & $16(21.4)$ \\
No & $58(77.3)$ \\
Unknown & $1(1.3)$ \\
Median duration of herniated disc & 30 days (range \\
Median duration of CES at presentation & 48 day-14 years) \\
$(n=68)$ & \\
& \\
&
\end{tabular}

* Total disc lesions: 79 (4 patients had double lesions: L4-L5+L5-S1 $(n=3)$ and L2-L3+L4-L5)

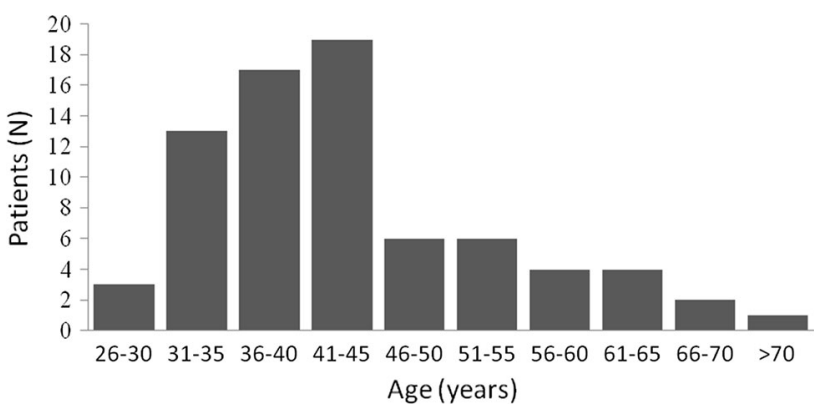

Fig. 1 Age at surgery. Distribution of age of CES patients at time of surgery

which did not exist before, which could be, e.g., incontinence or changed sensation of passing stool. A patient with fecal incontinence, since diagnosis of $M$ Sjogren several years before CES, was not classified as having complaints of defecation. Information about the presence of sexual dysfunction was available for 26 patients (19 men), of 


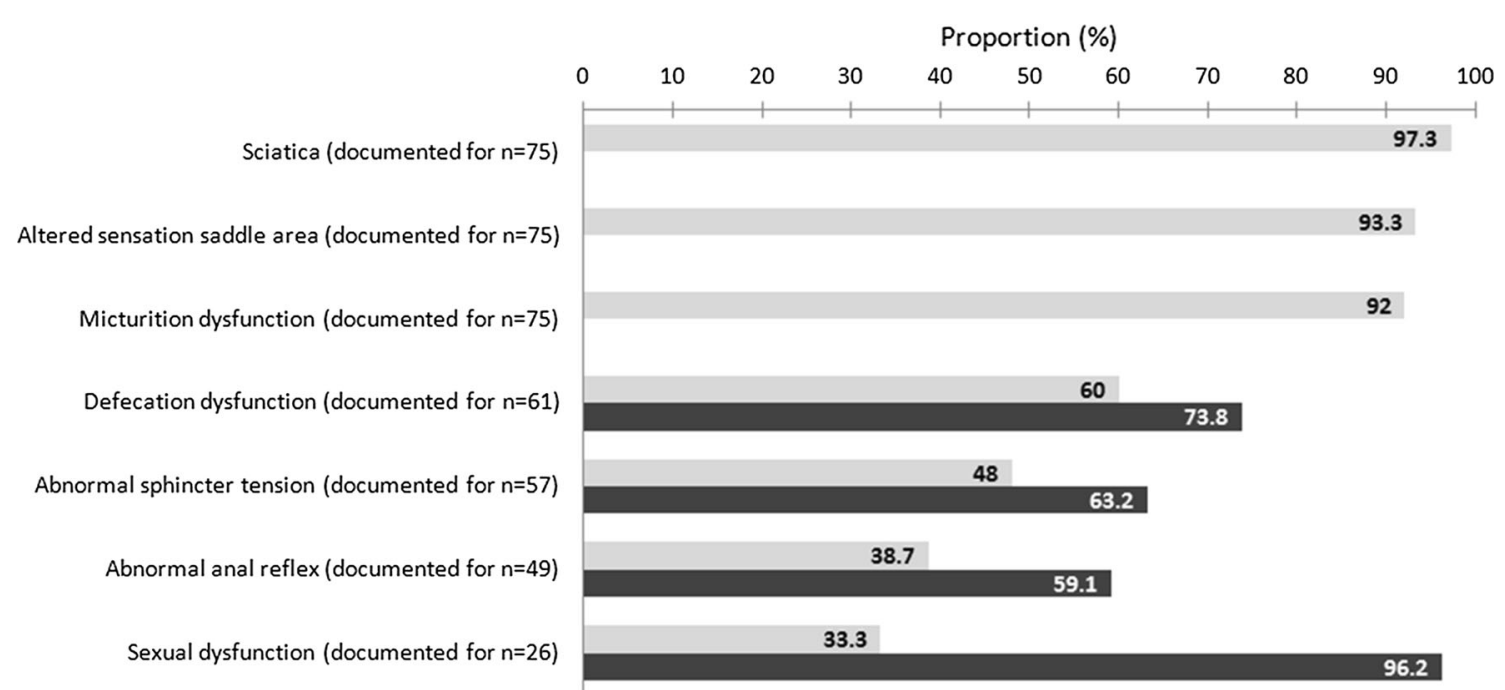

Fig. 2 CES at presentation. Prevalence of signs and symptoms of CES at presentation. The grey bars are the proportion of total patients included in this study $(n=75)$; the black bars are the proportion of patients for whom documentation was available ( $n$ specified after each row)

whom 25 experienced sexual dysfunction. Documented problems were, e.g., altered sensation of genitals, inability to reach orgasm, erectile dysfunction, and priapism. For two patients, sexual status (active/nonactive) before onset of CES was documented; for the others, no notes on sexual activity were found.

Anal sphincter tension and anal sphincter reflex (anal wink) were tested in 76.0 and $65.3 \%$ of patients, respectively, and were abnormal in the majority (63.2 and 59.1\%, respectively). Abnormal anal sphincter tension was significantly associated with altered sensation of the saddle area $(p=0.007$; Table 2$)$, with a sensitivity for altered sensation of the saddle area of $68 \%$. Abnormal anal sphincter reflex was not significantly associated with defecation dysfunction, although a trend was observed $(p=0.096$; Table 3$)$. Micturition dysfunction was not associated with either abnormal anal sphincter tension nor reflex (Table 4). Since data on sexual dysfunction at presentation were scarce, no analyses were done for sexual dysfunction.

Table 2 Association between altered sensation of the saddle area and sphincter tests. Association between defecation dysfunction and sphincter tests

\begin{tabular}{llll}
\hline & $\begin{array}{l}\text { Altered sensation } \\
\text { saddle }(\%)\end{array}$ & $\begin{array}{l}\text { Normal sensation } \\
\text { saddle }(\%)\end{array}$ & $p$ value \\
\hline $\begin{array}{c}\text { Abnormal anal } \\
\text { sphincter reflex }\end{array}$ & 60.9 & 33.3 & 0.347 \\
$\begin{array}{c}\text { Abnormal anal } \\
\text { sphincter tension }\end{array}$ & 68 & 0 & 0.007 \\
\hline
\end{tabular}

Table 3 Association between defecation dysfunction and sphincter tests. Proportion of patients with abnormal sphincter tests, stratified by defecation dysfunction (at presentation)

\begin{tabular}{llll}
\hline & $\begin{array}{l}\text { Defecation } \\
\text { dysfunction (\%) }\end{array}$ & $\begin{array}{l}\text { No defecation } \\
\text { dysfunction (\%) }\end{array}$ & $p$ value \\
\hline $\begin{array}{l}\text { Abnormal } \\
\text { anal sphincter } \\
\text { reflex }\end{array}$ & 64.5 & 33.3 & 0.096 \\
$\begin{array}{c}\text { Abnormal anal } \\
\text { sphincter tension }\end{array}$ & 63.9 & 58.3 & 0.731 \\
\hline
\end{tabular}

Proportion of patients with abnormal sphincter tests, stratified by defecation dysfunction (at presentation)

Table 4 Association between micturition dysfunction and sphincter tests. Proportion of patients with abnormal sphincter tests, stratified by micturition dysfunction (at presentation)

\begin{tabular}{llll}
\hline & $\begin{array}{l}\text { Micturition } \\
\text { dysfunction (\%) }\end{array}$ & $\begin{array}{l}\text { No micturition } \\
\text { dysfunction (\%) }\end{array}$ & $p$ value \\
\hline $\begin{array}{c}\text { Abnormal anal } \\
\text { sphincter reflex }\end{array}$ & 60.9 & 33.3 & 0.347 \\
$\begin{array}{c}\text { Abnormal anal } \\
\text { sphincter tension }\end{array}$ & 63.5 & 60 & 0.878 \\
\hline
\end{tabular}

Proportion of patients with abnormal sphincter tests, stratified by micturition dysfunction (at presentation)

\section{Association patient characteristics: presenting features}

The following factors were evaluated as predictors for presentation: age, gender, duration of complaints of herniated disc, duration of CES complaints, presence of altered sensation of the saddle area (for evaluating defecation and micturition dysfunction at presentation), 
presence of micturition dysfunction (for evaluating defecation dysfunction and altered sensation of the saddle area at presentation), and the presence of defecation dysfunction (for evaluating micturition dysfunction and altered sensation of the saddle area at presentation). For sciatica at presentation, the regression model could not be run, nor could sciatica at presentation be added as a predictor to the other models due to quasi-separation of the data. Defecation dysfunction at presentation was significantly associated with female gender (OR 4.11; $p=0.039$ ). Micturition dysfunction and altered sensation of the saddle at presentation displayed no predictors.

\section{Surgery}

All patients were decompressed by (partial) laminectomy and subsequent discectomy or sequesterectomy. A slight majority of patients $(n=36)$ was decompressed within $24 \mathrm{~h}$ after presentation (Fig. 3). Eight patients were decompressed more than $72 \mathrm{~h}$ after presentation at first doctor, with time to decompression of $96 \mathrm{~h}(n=3), 120 \mathrm{~h}$ $(n=1), 138 \mathrm{~h}(n=1), 168 \mathrm{~h}(n=1), 192 \mathrm{~h}(n=1)$, and $216 \mathrm{~h}(n=1)$. In 7 of these cases, majority of the delay was caused by the first doctor (family doctor or neurologist) where the patient presented. Hereafter, surgery was performed within $24 \mathrm{~h}(n=4), 48 \mathrm{~h}(n=2)$ and $72 \mathrm{~h}$ $(n=1)$ after first presentation at the neurosurgeon. In one case, no discrimination could be made between delay at first and second doctor.

\section{Post-operative outcome}

The first follow-up moment (FU 1) at which micturition, defecation, sexual function, and/or altered sensation of the saddle area was documented was on average $75 \mathrm{~h}$ postoperatively (range 4-336 h; median 48 h). The latest time

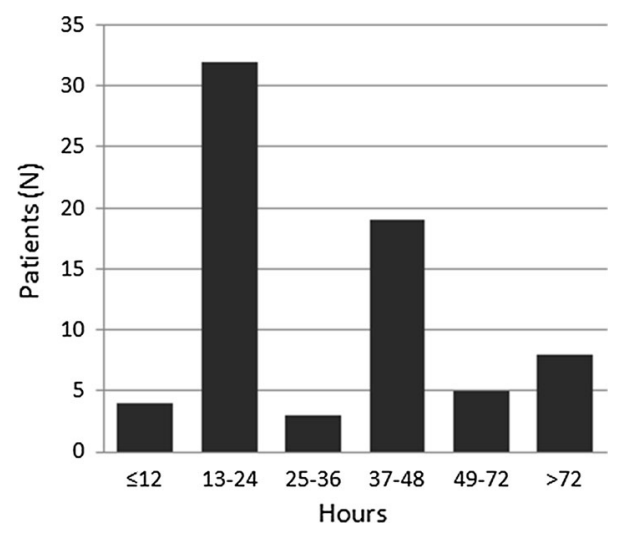

Fig. 3 Time to decompression. Distribution of time to decompression, counted from the first doctor visit due to CES complaints $(n=71)$ of FU 1 was 14 days post-operatively. Documentation on any of the items micturition, defecation, sexual function, and/or altered sensation of the saddle area at the second follow-up moment (FU 2) was available for 54 patients (72\%), with a mean FU time of 63 days (range 4-300 days, median 60 days).

A third follow-up moment (FU 3) at any item was documented for 23 patients $(31 \%)$, with a mean FU time of 265 days (range 56-730 days, median 225 days). FU 3 is reported in the text as corrected, not pooled, and is not used in any regression analysis.

\section{Micturition}

Documented micturition dysfunction decreased significantly comparing pre-operative moment with FU 2 (92.0 versus $47.7 \%, p<0.001)$, Fig. 4 . In one patient with postoperative dysfunction, requiring intermittent catheterization from the 4th day after surgery for a short period of time with complaints of urinary dysfunction up to the last follow-up moment, (6 months later), no complaints of micturition were documented at presentation. This was interpreted as misinformation at baseline. Pooled prevalence of micturition dysfunction was $47.7 \%$ at FU 2 . Reported dysfunction included: indwelling catheter, onoff catheterisation, suprapubic catheter, reduced feeling of passing urine, and (mild) incontinence. At FU 3, 19 patients were evaluated of whom 11 displayed dysfunction $(57.9 \%)$.

\section{Defecation}

Documented defecation dysfunction had decreased significantly after decompression measured at FU 2 (72.0 versus $41.8 \%, p=0.004)$, Fig. 5. For three patients, defecation dysfunction was documented post-operative but not pre-op. Pooled prevalence of dysfunction was $41.8 \%$ at FU 2.

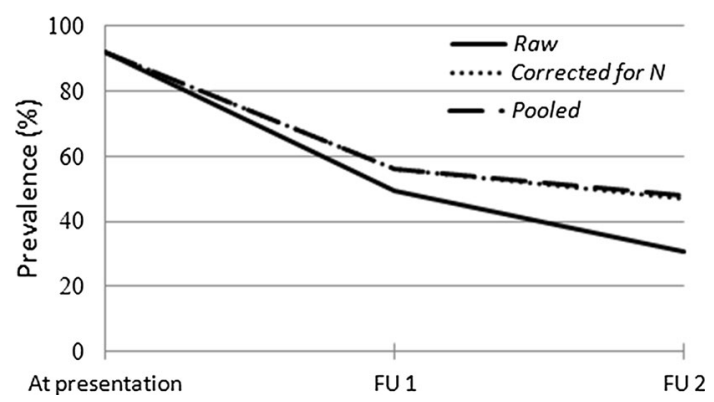

Fig. 4 Outcome of micturition dysfunction. The course of the proportion of patients with micturition dysfunction at presentation (documented for $n=75$ ), FU 1 (documented for $n=66$ ) and FU 2 (documented for $n=49$ ) 


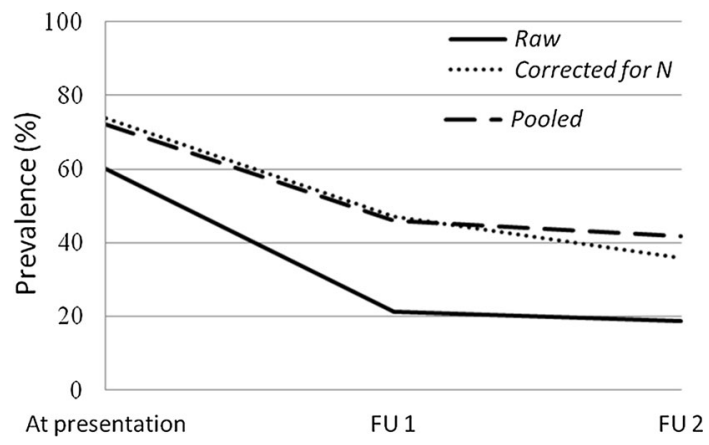

Fig. 5 Outcome of defecation dysfunction. The course of the proportion of patients with defecation dysfunction at presentation (documented for $n=61$ ), FU 1 (documented for $n=34$ ) and FU 2 (documented for $n=39$ )

Thirteen patients were evaluated at FU 3, of whom nine reported dysfunction $(69.2 \%)$.

\section{Sexual function}

Due to scarce data on sexual function, investigating that proportions were not done; therefore, no $p$ value for difference between pre- and post-operative dysfunction was derived. Corrected prevalence of sexual dysfunction at FU 2 was $53.3 \%$, Fig. 6. Documentation on sexual dysfunction was done for 5 patients at FU 3; four of them displayed dysfunction $(80 \%)$.

\section{Altered sensation of the saddle area and sciatica}

Documented altered sensation of the saddle area and sciatica both decreased significantly after decompression measured at FU 2 (93.3 versus 56.5\% and 97.3 and $47.5 \%$, respectively; both $p<0.001$ ), Figs. 7 and 8. At FU 3, 12 out of 18 patient with documentation reported altered sensation of the saddle area $(66.7 \%)$, and 12 out of 20 reported sciatica $(60 \%)$.

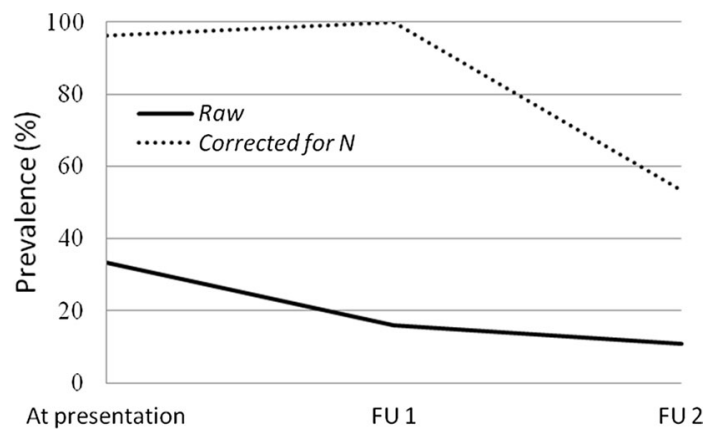

Fig. 6 Outcome of sexual dysfunction. The course of the proportion of patients with sexual dysfunction at presentation (documented for $n=26$ ), FU 1 (documented for $n=12$ ) and FU 2 (documented for $n=15$ ). Since multiple imputation was not used for data of sexual dysfunction, pooled data are not available

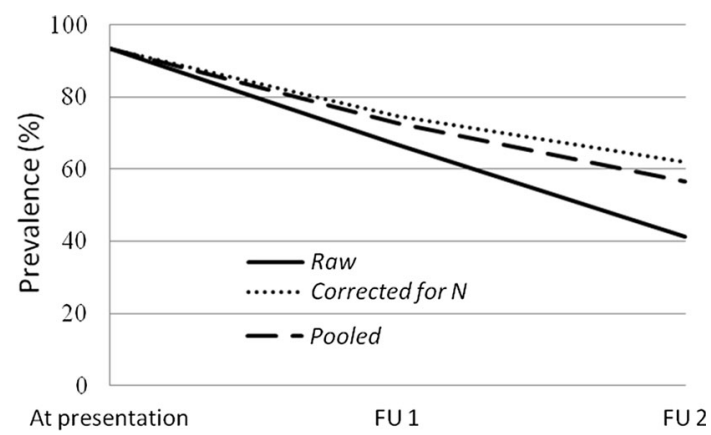

Fig. 7 Outcome of altered sensation of the saddle area. The course of the proportion of patients with altered sensation of the saddle area at presentation (documented for $n=75$ ), FU 1 (documented for $n=67$ ) and FU 2 (documented for $n=50$ )

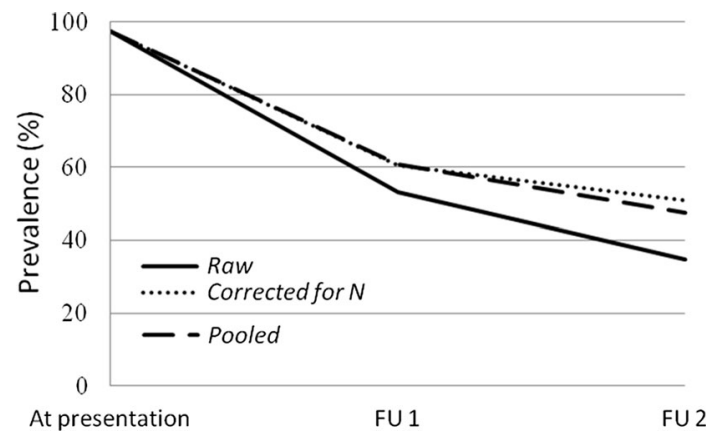

Fig. 8 Outcome of sciatica. The course of the proportion of patients with sciatica at presentation (documented for $n=73$ ), FU 1 (documented for $n=66$ ) and FU 2 (documented for $n=51$ )

\section{Predictors for outcome}

The following factors were evaluated as predictors for outcome: age, gender, duration of complaints of herniated disc, duration of CES complaints, time to decompression (stratified groups, see Methods section for details of groups), and altered sensation of the saddle area/micturition dysfunction/defecation dysfunction/sciatica at presentation.

Due to quasi-separated data, sciatica at presentation had to be removed from all models except the one for micturition dysfunction at FU 2; altered sensation of the saddle at presentation had to be removed from all models except the one for sciatica at FU 1; micturition dysfunction at presentation had to be removed from the models for sciatica at FU 2 and altered sensation of the saddle area at FU 1 and FU 2.

Timing of decompression was found to be significantly associated with short-term outcome (FU 1) of sciatica: less time to decompression was associated with more sciatica at FU $1(p=0.042)$. After stratifying outcomes for time to decompression using the well-known break points from literature of $48 \mathrm{~h}[12,14]$ and $36 \mathrm{~h} \mathrm{[13],} \mathrm{we} \mathrm{found} \mathrm{a} \mathrm{sta-}$ tistically significant difference for outcome of sciatica at 
FU 1 comparing decompression before and after $36 \mathrm{~h}$. Patients decompressed within $36 \mathrm{~h}$ experienced more sciatica than patients decompressed after $36 \mathrm{~h}$ (79.4 versus $37.9 \%$, adjusted $p=0.032$ ). There was no difference comparing patients being decompressed before and after $48 \mathrm{~h}$. There was no association between timing to decompression and outcome of sciatica at FU 2 ( $p=0.475$ ). No other statistically significant predictors for micturition, defecation, altered sensation of the saddle area, and sciatica at FU 1 and FU 2 were identified.

\section{Discussion}

This is the largest single study performed about outcome of micturition, defecation, and sexual function in CES $(n=75)$. The incidence of CES among patients being operated on for herniated disc was relatively high in this study: $10.8 \%$ compared to $1-3 \%$ in literature [9, 17]. This high incidence can be explained by the fact that the LUMC serves as a referral hospital for urgent neurosurgical cases. The CES definition that was used to include patients in this study is widely used in literature, and even though a univocal definition for CES does not exist, the authors believe that using this definition guaranteed a fair representation of CES patients. This study displays unique data on the presenting symptoms of a large group of CES patients, proving that, next to the well-acknowledged micturition dysfunction, also defecation and sexual dysfunction are common at presentation.

Anal sphincter tension and anal sphincter reflex are often tested in CES patients, even though several studies found no diagnostic value for these tests [18-21]. In the current study, abnormal anal sphincter reflex was not significantly associated with any of the diagnostic criteria for CES used in this study (closest to an association was defecation dysfunction with $p=0.096$ ). Abnormal anal sphincter tension is significantly associated with altered sensation of the saddle area $(p=0.007)$. Specificity of the test is $100 \%$ (all patients without altered sensation of the saddle area displayed normal sphincter tension) and sensitivity is $68 \%$ (a substantial proportion of patients with anamnestic altered sensation of the saddle area displayed normal sphincter tension). With a positive predictive value of $100 \%$ (all patients with abnormal sphincter tension had anamnestic altered sensation of the saddle area) and a negative predictive value of merely $19 \%$, abnormal anal sphincter tension at physical examination supports the presence of altered sensation of the saddle area, but in no way rules out altered sensation of the saddle area, in case it is normal. Considering a specificity of $100 \%$, the question arises whether it is necessary to test sphincter tension in patients without altered sensation of the saddle area, since it might cause unnecessary discomfort. Evaluating the anal sphincter reflex data, however, does indicate that sphincter testing might add extra information. Of the five patients with normal sensation of the saddle area at presentation, four were tested for anal sphincter tension (all normal). Two of those four were also tested for the anal sphincter reflex, which in one case was abnormal, demonstrating that with normal sensation of the saddle area and with normal sphincter tension, the anal sphincter reflex can still be abnormal. It could, therefore, be sensible to do anal sphincter reflex tests even in a patient with normal sensation of the saddle area. The fact that only a small proportion in our study sample demonstrated normal sensation of the saddle are at presentation $(n=5)$, limits our data in this aspect and, therefore, clinical relevance of sphincter tests cannot be secured nor refuted based on those findings.

Prevalence of micturition dysfunction is $47.7 \%$ of patients at FU 2. In an older study discussing 13 patients with CES due to herniated disc, the author states that "in all such patients, there was incomplete return of normal micturition" [22]. In a more recent study of McCarthy et al., a better recovery of micturition has been described: of 42 evaluated patients with CES due to herniated disc, $36 \%$ reported urinary incontinence (mean FU time of 60 months) [23]. The higher prevalence of micturition dysfunction in the current study might be due to the shorter FU time, assuming that function of micturition will improve gradually over time.

Regarding defecation dysfunction, this study found a prevalence of $41.8 \%$ at FU 2; McCarthy et al. found a higher prevalence at follow-up (60\%): this could be due to attrition bias, the chance of which becomes greater when follow-up period of the study is longer-as in McCarthy's study. Sexual dysfunction in the current study was $53.3 \%$ at FU 2, which is quite similar to the prevalence of $57 \%$ reported by McCarthy et al. The true prevalence of sexual dysfunction might be higher than the ones found in studies; since the threshold to discuss sexual health is very high which, it is unlikely that either doctor or patient opens the topic, even if there are complaints.

Two patients in this study displayed a specific feature of sexual dysfunction: priapism. One patient reported spontaneous erections at presentation; unfortunately, no documentation on sexual function was done at follow-up for this patient. The second patient presented with a numb feeling of the penis which had changed to priapism at follow-up after 7 weeks and after 5.5 months. Priapism as a feature of cauda equina compression is extremely uncommon and only two reports in literature describe such a case: one involves a 61-year-old man with a herniated disc at L4-L5 [24], the other a 60-year-old man with a degenerative stenosis at L3-L4 and lumbar arachnoiditis [25]. Both patients experienced priapism and a numb, respectively, a 
burning sensation at the saddle area when walking, without sphincter disturbances. After decompression of the cauda equina, both patients experienced immediate and complete relieve of their symptoms, suggesting a causal relationship of cauda equina claudication and priapism. The parasympathetic fibers that are responsible for penile erection arise in S2-S4, and it is thought that their stimulation through (in these two cases: intermittent) compression had resulted in priapism [24]. To the authors' best knowledge, there are no case reports about priapism in nonintermittent cauda equina compression. Even though the course of priapism complaints of the two patients in this study is uncertain, the authors believe that it is not unthinkable that the priapism experienced by the two patients in this study might be attributed to compression of the cauda equina, even though exact mechanisms remain unclear.

This study found that female patients are more likely to present with defecation dysfunction than their male counterparts (OR 4.11; $p=0.039$ ). Epidemiologic studies demonstrate that female gender is associated with more constipation, as a baseline fact in the general population as well as in the CES population when focusing on outcome [26, 27].

Sciatica were more often present at FU 1 when time to decompression was shorter (OR 1.86; $p=0.042$ ), which association had disappeared at FU 2. This association nowise undermines the importance of emergency decompression. It rather displays a correlation between duration of compression and other (prognostic) factors for which could not be corrected. Those factors are (1) the speed with which the compression has arisen, stating that patients with slowly developing anatomical lesions have a more favourable prognosis [28, 29] and (2) the type of CES lesion, stating that a "complete" CES (with total obstruction) carries a poorer prognosis than a "incomplete" CES, adding that the incomplete type often occurs more gradually, (although not exclusively) [22, 30]. It seems sensible that a patient with sudden onset of heavy symptoms in general presents earlier. This results in a shorter duration of CES complaints (patient delay) as well as a shorter time to decompression (doctor delay), even though outcome is poor (due to the extent of the lesion). Patients that show reasonable outcome when decompression is delayed by weeks [31], probably had a favourable anatomical lesion and more gradual onset of complaints. Since it is not (yet) possible to distinguish the group with the favourable conditions from the group with the less favourable conditions at the time of presentation (since, e.g., the exact correlation of clinical presentation and degree of canal obstruction on imaging is not yet known), it is necessary to decompress every CES patient as soon as possible, to create the best chances for fair recovery.

The authors strongly believe that this study's finding, e.g., that time to decompression is not associated with outcome of micturition, defecation, and altered sensation of the saddle area, and does display a correlation of more sciatica at FU1 when it is shorter, does not implicate that decompression in CES is to be delayed. First, the number of patients with delayed decompression in this study is relatively small (eight patients in the group decompressed after $72 \mathrm{~h}$; five patients in the group decompressed within 48-72 h). Previously, meta-analyses have displayed better outcomes with decompression taking place within $48 \mathrm{~h}$ [12, 14] or within $36 \mathrm{~h}$ [13]. Some studies with smaller patient numbers display a significant better outcome after earlier decompression as well, with significant better outcomes with decompression within $48 \mathrm{~h}$ [9] and even after 10 days [4]. Studies displaying no difference in outcome are a minority and evaluate relative small patient numbers only [10, 11]. The finding in this study that a shorter time to decompression is associated with more sciatica at FU 1, should, therefore, be weighed by the small patient number of this study and the fact that this finding is not present at FU 2 anymore. In addition, in this study, clinical motivesunknown to the authors due to the retrospective study design_could have led to the decision for very early decompression in specific patient groups, which might have caused selection bias. The outcome would then be more influenced by factors on which clinical motives are based (and which have led to an early time to decompression) than by the actual time to decompression.

Significant predictors, such as duration, of CES complaints for more than $48 \mathrm{~h}$ as a risk factor for micturition dysfunction [32] and defecation dysfunction at presentation as a risk factor for sexual dysfunction [23] could not be identified in this study.

Missing data in this study are partly attributed to the inclusion of patients that were referred for surgery to LUMC, and were seen for follow-up at their original referring hospital (in which case the researchers did not have access to the follow-up data). Of the 19 patients that originated from either LUMC or the general practitioner, $100 \%$ displayed data at FU 2. Of the 55 patients that originated from a different hospital and were referred to LUMC for surgery, 34 patients $(61.8 \%)$ displayed data at FU 2. The amount of dysfunction between the group of LUMC patients and the group originating from a different hospital and referred to LUMC was not significantly different at FU 2: neither for micturition dysfunction (42.1 versus $48.3 \% ; p=0.771)$ nor for defecation dysfunction (23.5 versus $45.5 \% ; p=0.193)$, sciatica (57.9 versus $46.9 \% ; p=0.565)$, or altered sensation of the saddle area (58.8 versus $63.6 \%$; $p=0.767$ ). This reason for missing data was, therefore, not believed to have caused bias.

Apart from loss to follow up of patients belonging to a different original hospital, three other reasons for missing data in this retrospective design are: (1) the topic was not 
discussed and, therefore, no notes are available, (2) the topic was discussed, but no notes were taken, and (3) the patient did not show up at FU moment. Especially for the latter two reasons, data are more likely to be missing when there are no complaints. To explore the extent of this bias-i.e., the hypothesis that data of patients with no complaints are more prone to be missing-patients with and patients without documented complaints at the previous check-up were analyzed for the amount of available data (Figs. 9, 10, 11, 12, 13). As is displayed, patients without documented complaints at their previous check-up are more prone to have missing data (apart from the case of defecation dysfunction, which cannot be easily explained). Due to this fact, the authors have chosen to not use FU 3 as main outcome parameter, even more since FU 3 is more likely to be planned for patients with complaints, therefore, contributing substantially to attrition bias when it would be used as a main outcome parameter.

Data on sexual function in this study were particularly scarce, as well at presentation as during follow-up, which resulted in the inability of performing several analyses

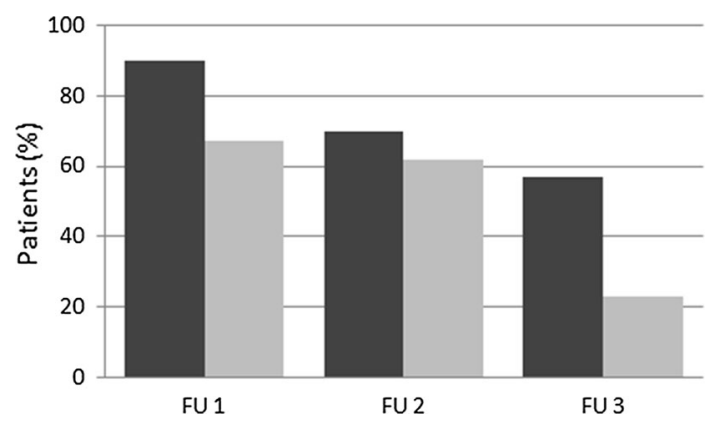

Fig. 9 Availability of data on micturition. The proportion of available data per FU moment is stratified by patients for whom complaints were documented at the previous FU moment (black bar) and by patients for whom it was documented that there were no complaints at the previous FU moment (grey bar)

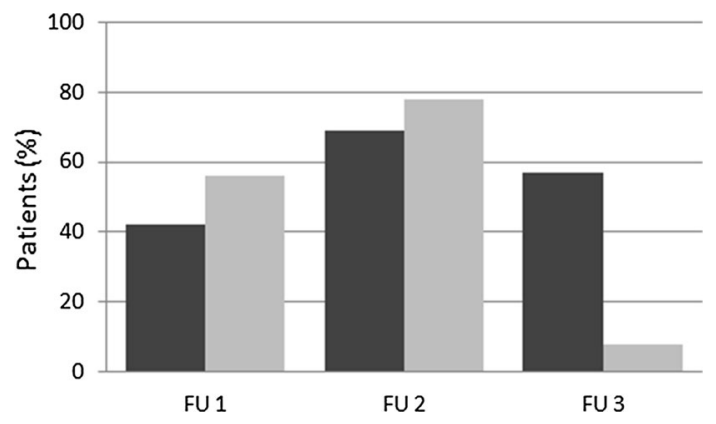

Fig. 10 Availability of data on defecation. The proportion of available data per FU moment is stratified by patients for whom complaints were documented at the previous FU moment (black bar) and by patients for whom it was documented that there were no complaints at the previous FU moment (grey bar)

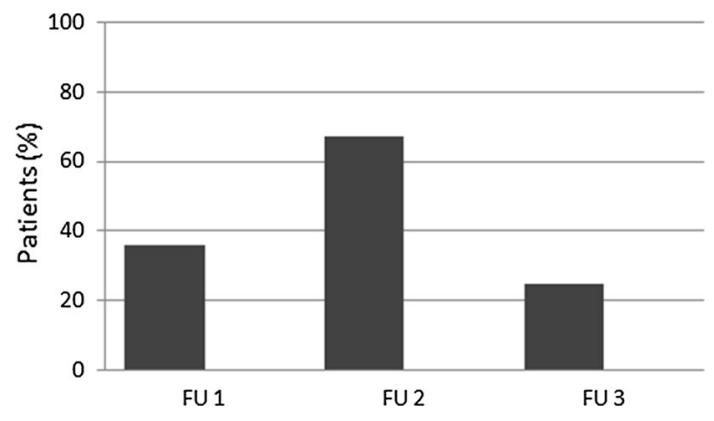

Fig. 11 Availability of data on sexual function. The proportion of available data per FU moment is shown for patients for whom complaints were documented at the previous FU moment (black bar). For all patients for whom it was documented that there were no complaints at the previous FU moment, there was no data available at the next FU moment; therefore, there are no grey bars

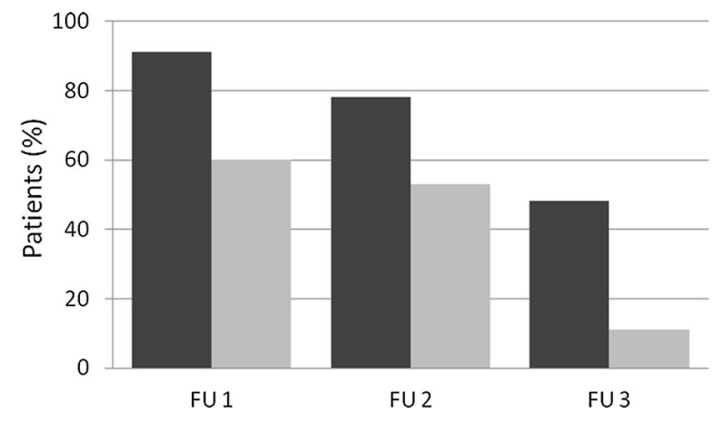

Fig. 12 Availability of data on sensation of the saddle area. The proportion of available data per FU moment is stratified by patients for whom complaints were documented at the previous FU moment (black bar) and by patients for whom it was documented that there were no complaints at the previous FU moment (grey bar)

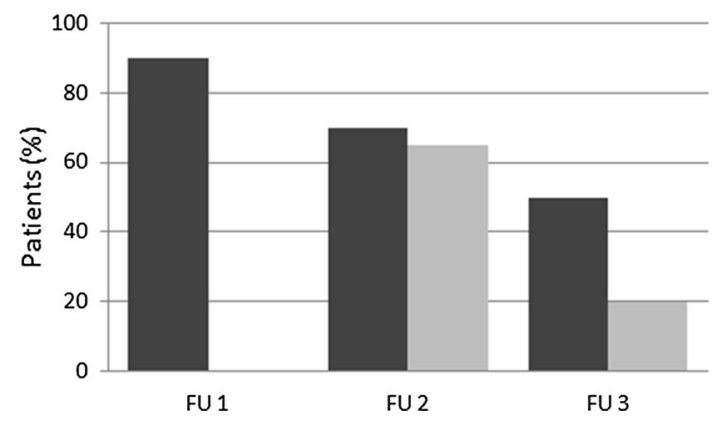

Fig. 13 Availability of data on sciatica. The proportion of available data per FU moment is stratified by patients for whom complaints were documented at the previous FU moment (black bar) and by patients for whom it was documented that there were no complaints at the previous FU moment (grey bar). For all patients for whom it was documented that there were no complaints at presentation, there were no data available at FU 1; therefore, there is no grey bar at FU 1

regarding sexual dysfunction. Limited data on sexual function are ubiquitous in CES patients in literature [2], most likely due to barriers on both the patients' side as well as on the doctor's side, which could unfortunately not be minimized in this retrospective study design. The nature of 
the available data on sexual dysfunction at presentation is striking: 25 out of 26 patients experienced sexual dysfunction. The fact that sexual function is more often documented for male patients than for their female counterparts is something that is believed by the authors to be due to both patient factors as well as doctor factors [15].

\section{Conclusion}

This is the largest single study about outcome in CES after decompression. It displays unique data about the outcome of micturition, defecation, sexual function, sciatica, and altered sensation of the saddle area, as well as presenting features of a large cohort of CES patients, proving that micturition, defecation, and sexual dysfunction are common at both presentation and at follow-up. Female patients were found to have significant more defecation dysfunction at presentation than their male counterparts. A shorter time to decompression was a risk factor for sciatica shortly after surgery (at follow-up moment 1), but not for long-term outcome. Studies on correlation between imaging at presentation and outcome could help identifying the patients being more at risk at presentation. Missing data were handled with multiple imputation with analysis of possible bias. A follow-up study is recommended for more longterm follow-up data. In conclusion, recovery after decompression for CES does take a long time and is not complete in a substantial amount of cases; something for which we should adequately prepare our patients when diagnosing CES.

\section{Compliance with ethical standards}

\section{Conflict of interest None.}

Open Access This article is distributed under the terms of the Creative Commons Attribution 4.0 International License (http://crea tivecommons.org/licenses/by/4.0/), which permits unrestricted use, distribution, and reproduction in any medium, provided you give appropriate credit to the original author(s) and the source, provide a link to the Creative Commons license, and indicate if changes were made.

\section{References}

1. Fraser S, Roberts L, Murphy E (2009) Cauda equina syndrome: a literature review of its definition and clinical presentation. Arch Phys Med Rehabil 90(11):1964-1968

2. Korse NS, Jacobs WC, Elzevier HW, Vleggeert-Lankamp CL (2013) Complaints of micturition, defecation and sexual function in cauda equina syndrome due to lumbar disk herniation: a systematic review. Eur Spine J 22(5):1019-1029

3. Mixter WJ, Barr JS (1934) Rupture of the intervertebral disk with involvement of the spinal canal. N Engl J Med 211(5):210-225
4. Busse JW, Bhandari M, Schnittker JB, Reddy K, Dunlop RB (2001) Delayed presentation of cauda equina syndrome secondary to lumbar disk herniation: functional outcomes and health related quality of life. CJEM 3(4):285-291

5. Dinning TA, Schaeffer HR (1993) Discogenic compression of the cauda equina syndrome: a surgical emergency. Aust N Z J Surg 3(12):927-934

6. Nielsen B, de Nully M, Schmidt K, Hansen RI (1980) A urodynamic study of cauda equina syndrome due to lumbar disk herniation. Urol Int 35(3):167-170

7. O'Laoire SA, Crockard HA, Thomas DG (1981) Prognosis for sphincter recovery after operation for cauda equina compression owing to lumbar disc prolapsed. BMJ 282:1852-1854

8. Shapiro S (1993) Cauda equina syndrome secondary to lumbar disk herniation. Neurosurgery 32(5):743-746 (discussion 746-747)

9. Shapiro S (2000) Medical realities of cauda equina syndrome secondary to lumbar disc herniation. Spine 25:348-351

10. Olivero WC, Wang HA, Hanigan WC, Henderson JP, Tracy PT, Elwood PW et al (2009) Cauda equina syndrome (CES) from lumbar disk herniations. J Spinal Disord Tech 22(3):202-206

11. Qureshi A, Sell P (2007) Cauda equina syndrome treated by surgical decompression: the influence of timing on surgical outcome. Eur Spine J Dec 16(12):2143-2151

12. Ahn UM, Ahn NU, Buchowski JM, Garrett ES, Sieber AN, Kostuik JP (2000) Cauda equina syndrome secondary to lumbar disc hernation: a meta-analysis of surgical outcomes. Spine 25:1515-1522

13. DeLong WB, Polissar N, Neradilek B (2008) Timing of surgery in cauda equine syndrome with urinary retention: meta-anlysis of observation studies. J Neursurg Spine 8(4):305-320

14. Todd NV (2005) Cauda equine syndrome: the timing of surgery probably does influence outcome. Br J Neurosurg 19(4):301-306

15. Korse NS, Nicolai MP, Both S, Vleggeert-Lankamp CL, Elzevier HW (2016) Discussing sexual health in spinal care. Eur Spine J 25(3):766-773

16. Korse NS, Nicolai MP, Both S, Vleggeert-Lankamp CL, Elzevier HW (2016) Discussing reproductive health in spinal care, part II: fertility issues. Eur Spine J 25(9):2945-2951

17. Gleave JR, Macfarlane R (2002) Cauda equine syndrome: what is the relationship between timing of surgery and outcome? $\mathrm{Br} \mathrm{J}$ Neurosurg 16:325-328

18. Ahad A, Elsayed M, Tohid H (2015) The accuracy of clinical symptoms in detecting cauda equina syndrome in patients undergoing acute MRI of the spine. Neuroradiol J 28(4):438-442

19. Balasubramanian K, Kalsi P (2010) Greenough CG (2001) Reliability of clinical assessment in diagnosing cauda equina syndrome. Br J Neurosurg 24(4):383-386

20. Domen PM, Hofman PA, van Santbrink (2009) Predictive value of clinical characteristics in patients with suspected cauda equina syndrome. Eur J Neurol 16:416-419

21. Gooding BWT, Higgins MA, Calthorpe DAD (2013) Does rectal examination have any value in the clinical diagnosis of cauda equina syndrome? Br J Neurosurg 27(2):156-159

22. Shephard RH (1959) Diagnosis and prognosis of cauda equina syndrome produced by protrusion of lumbar disk. Br Med J 2(5164):1434-1439

23. McCarthy MJ, Aylott CE, Grevitt MP (2007) Cauda equina syndrome: factors affecting long-term functional and sphincteric outcome. Spine 32:207-216

24. Ravindran M (1979) Cauda equina compression presenting as spontaneous priapism. J Neurol Neurosurg Psychiatry 42:280-282

25. Maurice-Williams RS, Marsh HT (1985) Priapism as a feature of claudication of the cauda equina. Surg Neurol 23:626-628 
26. Johanson JF, Sonnenberg A, Koch TR (1989) Clinical epidemiology of chronic constipation. J Clin Gastroenterol 11(5):525-536

27. Podnar S (2006) Bowel dysfunction in patients with cauda equina lesions. Eur J Neurol 13:1112-1117

28. Gleave JR, MacFarlane R (1990) Prognosis for recovery of bladder function following lumbar central disc prolapse. $\mathrm{Br} \mathrm{J}$ Neurosurg 4(3):205-209

29. Nascone JW, Lauerman WC, Wiesel SW (1999) Cauda equina syndrome: is it a surgical emergency? Univ Pennsyl Orthop J $12: 73-76$
30. Gardner A, Gardner E, Morley T (2011) Cauda equina syndrome: a review of the current clinical and medico-legal position. Eur Spine J 20(5):690-697

31. Dhatt S, Tahasildar N, Tripathy SK, Bahadur R, Dhillon M (2011) Outcome of spinal decompression in cauda equine syndrome presenting late in developing countries: case series of 50 cases. Eur Spine J 20(12):2235-2239

32. Aho AJ, Auranen A, Pesonen K (1969) Analysis of cauda equina symptoms in patients with lumbar disk prolapse. Pre-operative and follow-up clinical and cystometric studies. Acta Chir Scand 135(5):413-420 